\title{
METAPHASE I BOUND ARMS AND CROSSING OVER FREQUENCY IN RYE. IV. TRITICALE-RYE HYBRIDS
}

\author{
JUAN ORELLANA AND RAMON GIRALDEZ* \\ Departamento de Genética, Facultad de Biología, Universidad Complutense, Madrid, Spain; \\ * Departmento de Genética, Facultad de Biología, Universidad de Oviedo, Oviedo, Spain \\ Received 9.ix.83
}

\section{SUMMARY}

\begin{abstract}
The correspondence between the number of metaphase I associations (bonds) and the chiasma frequency of specific chromosome arms of rye has been analysed in the meiosis of Triticale-rye hybrid plants. These plants were heterozygous for prominent telomeric $\mathrm{C}$-bands in the two arms of chromosome $1 \mathrm{R}$. From the frequency of bound arms at metaphase I and the frequency of recombined chromosome at anaphase I in the arms considered, estimates of chiasma frequency can be made under the assumption that at least one chiasma per bond was formed. However, these estimates lead to the absurdity of negative chiasma frequencies. The discrepancy between metaphase I and anaphase I results, due to an excess of anaphase I parental chromosomes, can be explained on the assumption that some of the bonds appearing at metaphase I are achiasmate and a remnant of prophase pairing.
\end{abstract}

\section{INTRODUCTION}

In Triticinae, the number of chiasmata cannot be determined at diplotene. At metaphase I, often only associations (bonds) between bivalent arms can be observed, these bonds being interpreted traditionally as an indication of the presence of at least one chiasma.

In previous studies (Giraldez and Orellana, 1979; Orellana and Giraldez, $1981,1983)$ a procedure was used for estimating chiasma frequency in specific chromosome arms of rye marked with Giemsa banding in meiosis. Plants differing in respect to the C-banding pattern of specific chromosome arms were used. From the frequency of being bound at metaphase $I$ and the frequency of recombinant chromosomes at anaphase I or metaphase II in the arms considered, estimates of chiasma frequencies were derived.

The materials employed in those instances were an open pollinated variety of rye (Orellana and Giraldez, 1981), the F1s between rye inbred lines (Giraldez and Orellana, 1979; Orellana and Giraldez, 1981) and partially desynaptic plants obtained from the backcrosses between those F1s and their parental inbred lines (Orellana and Giraldez, 1983).

The results indicated that more than one chiasma per arm could be formed in some chromosomes of the cultivar and the F1s. However, in desynaptic plants an excess of anaphase I parental type chromosomes was found, being interpreted as produced by the existence of non-chiasmate bonds, i.e., some of the bonds appearing at metaphase I were actually a remnant of prophase pairing, no chiasmata being formed between the arms involved.

In the present work, the analysis has been extended to chromosome $1 R$ in Triticale-rye hybrids. The existence of metaphase I $1 R$ bivalents having no chiasmata is demonstrated. 


\section{Material and Methods}

Four Triticale-rye hybrid plants (genome constitution ABRR) obtained from the cross between a hexaploid Triticale (cv. Cachirulo) and a rye inbred line (Line E) formed the material for this study.

In order to obtain mitotic metaphase cells, seeds were germinated on wetted filter paper in Petri dishes at room temperature. When primary roots were $1 \mathrm{~cm}$ long they were excised and immersed in tap water at $0^{\circ} \mathrm{C}$ for $24 \mathrm{~h}$ to shorten the chromosomes. Subsequently, the tips were fixed in acetic alcohol $1: 3$. For meiotic cells, anthers were fixed in acetic alcohol $1: 3$.

Both root tips and anthers were maintained in the fixative during one to four months at $3-4^{\circ} \mathrm{C}$. The fixed material was squashed and stained following the Giensa $\mathrm{C}$-banding technique described previously (Giraldez et al., 1979).

\section{REsUlts}

Plates 1(a)-(b) show C-banded mitotic metaphases of the Triticale (cv. Cachirulo) and rye inbred line plants used in the cross from which the Triticale-rye hybrids were obtained. There are differences in the C-banding pattern of most rye chromosomes, those of Triticale having a higher amount of telomeric C-heterochromatin than the ones of inbred line $\mathrm{E}$.

Using the C-banding technique it was possible to identify chromosome pair $1 \mathrm{R}$ in the meiosis of the Triticale-rye hybrids analyzed. As expected, these plants were heterozygous for the C-banding pattern of this chromosome pair. Chromosome $1 \mathrm{R}$ proceeding from Triticale had C-heterochromatin blocks in both telomeres whereas the one proceeding from inbred line $\mathrm{E}$ had almost no telomeric $\mathrm{C}$-heterochromatin.

Plates 2(a)-(b) show some examples of metaphase I C-banded cells of the Triticale-rye hybrid plants studied.

Table 1 shows the three types of middle metaphase I chromosome pairing observed (rye-rye, wheat-wheat and wheat-rye) indicating the frequencies of the different configurations for chromosome pair $1 R$ ( $R$, ring bivalents; $\mathrm{O} 1$, open bivalents with the long arm bound; Os, open bivalents with the short arm bound; $U$, univalents) and for the rest of rye and wheat chromosomes ( $R$, ring bivalents; $O$, open bivalents; $U$, univalents).

It is worth mentioning that, at metaphase $I$, univalents and not bound arms of chromosomes forming open bivalents showed no evidence of recombination, i.e., the two chromatids of these chromosome arms showed the same banding pattern.

On the other hand, all wheat-rye bivalents were open, the two $1 \mathrm{R}$ chromosomes being involved with almost the same frequency. Wheat-rye trivalents were formed by two wheat and one rye chromosome. In all wheat-rye associations of chromosome $1 \mathrm{R}$ only long arm was involved.

A similar conclusion to that obtained by Naranjo (1982) can be deduced from table 1 . There is a preferential occurrence of wheat-rye meiotic pairing between chromosomes of homoeologous group 1 , in spite of the fact that in rye-rye associations the average number of bound arms was lower in pair $1 \mathrm{R}$ than in the remaining rye chromosome pairs. This suggests a greater affinity or a more efficient pairing between wheat and rye chromosomes for homoeologous group 1. 

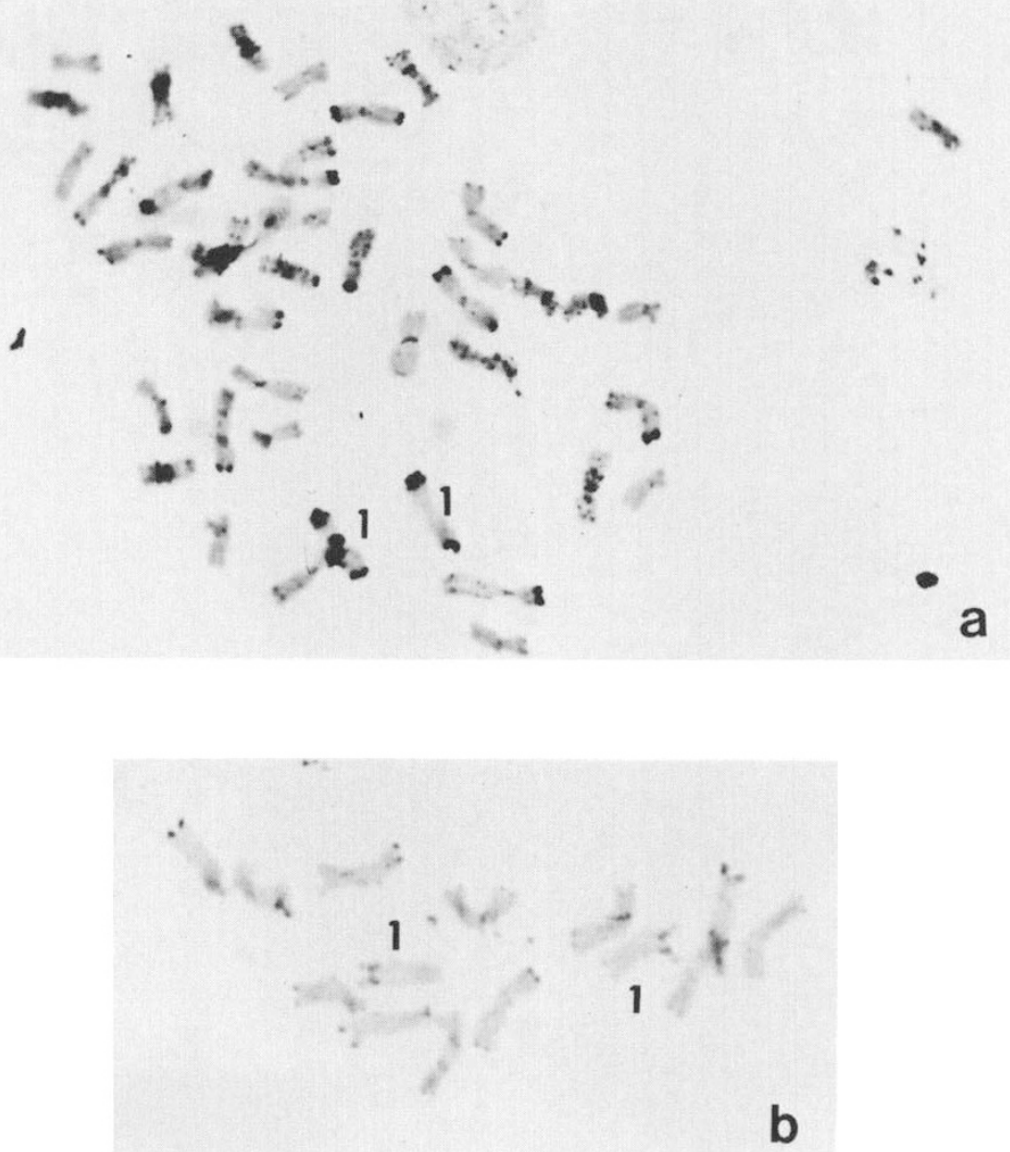

Plate 1. C-banded somatic metaphase cells. (a) Plant of the hexaploid Triticale cv. Cachirulo. (b) Plant of inbred line $\mathrm{E}$ of rye. 


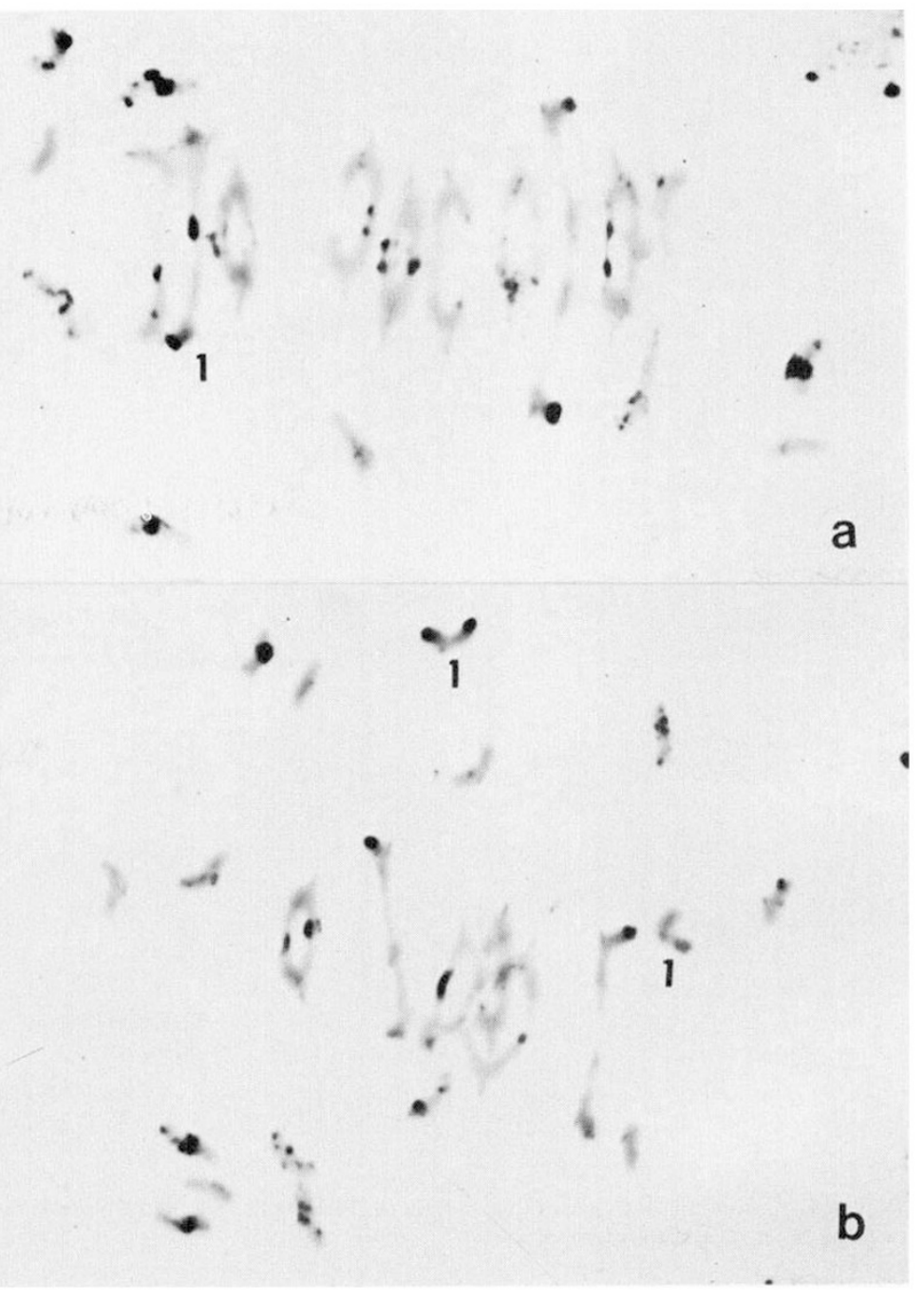

Plate 2. C-banded metaphase I cells of the Triticale $\times$ rye hybrids. (a) The $1 \mathrm{R}^{\mathrm{L}}$ chromosome arm is bound. (b) Both arms of chromosome $1 \mathrm{R}$ are unbound. 


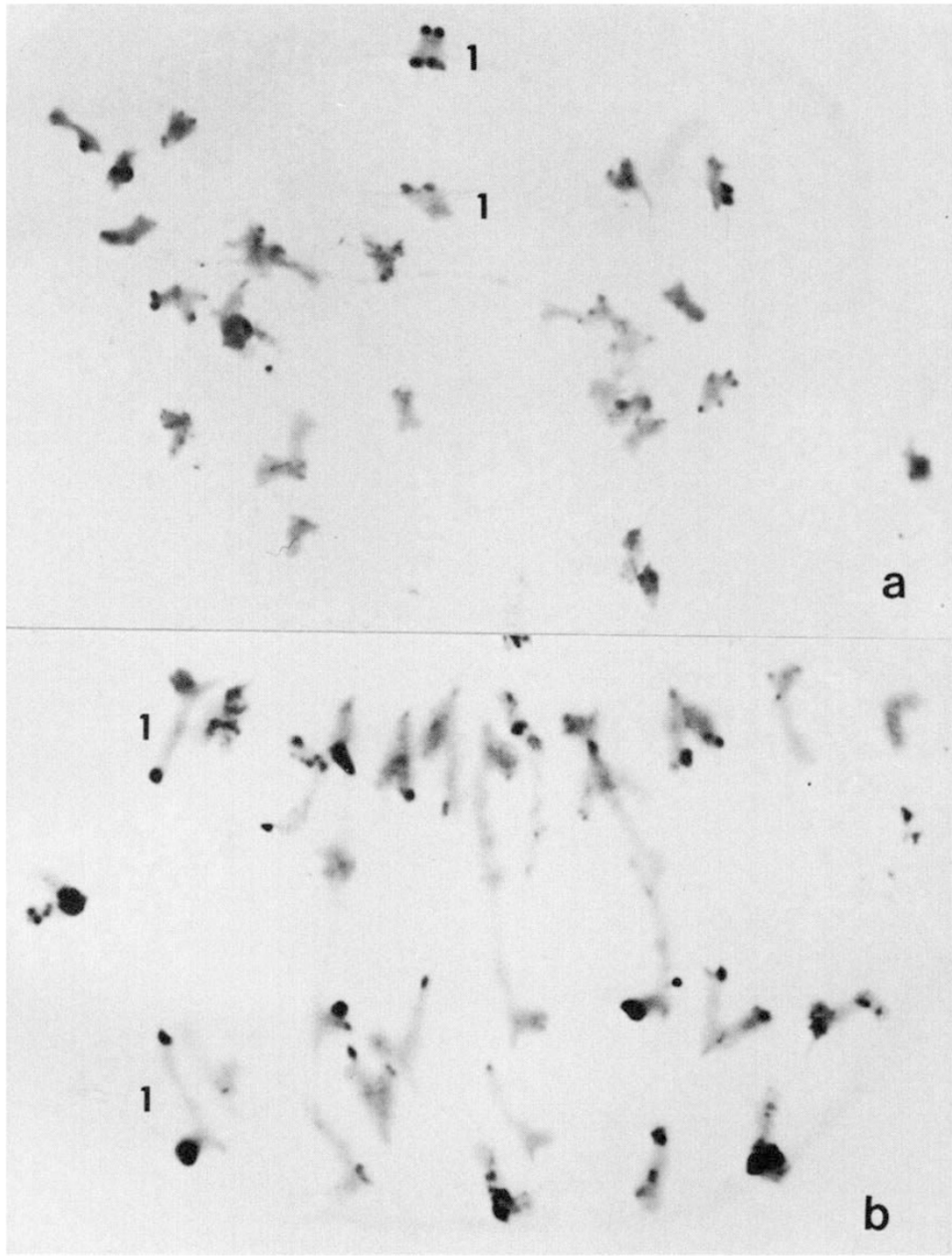

Plate 3. C-banded anaphase I cells of the Triticale $\times$ rye hybrid plants. (a) Parental type for both $1 \mathrm{R}$ chromosome arms. (b) Recombinant type for $1 \mathrm{R}^{\mathrm{L}}$ chromosome arm and parental type for $1 R^{S}$ chromosome arm. 


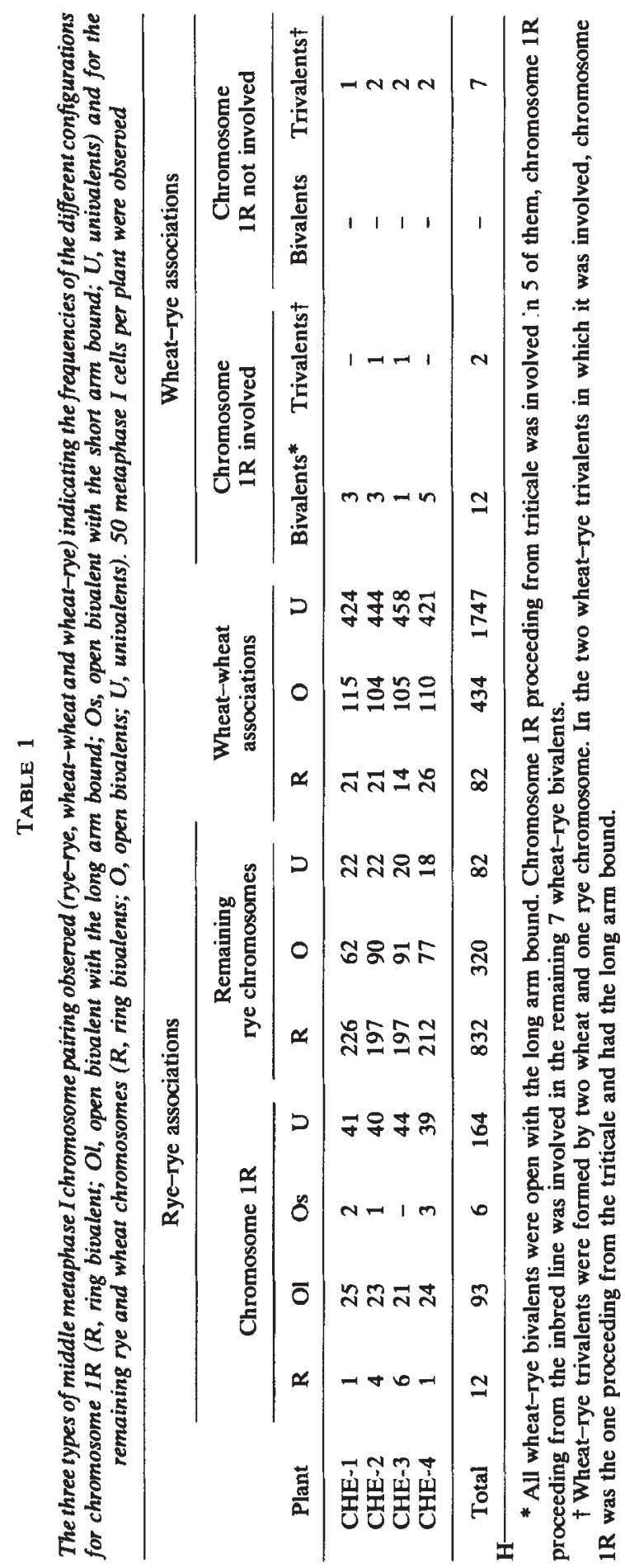




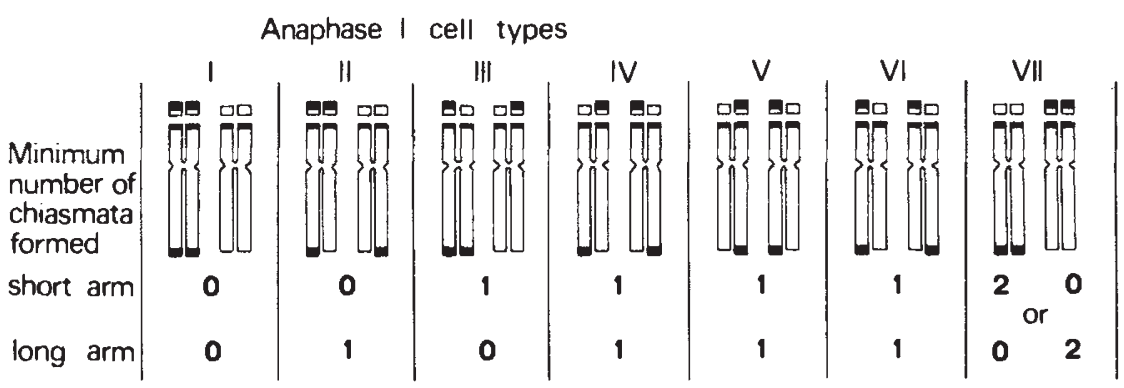

F1G. 1. The different metaphase I cell types expected in respect to the constitution of their chromosome $1 \mathrm{R}$ (showing or not evidence of recombination in the two arms) and the minimum number of chiasmata necessary for their appearance.

As chromosome pair 1R was heterozygous for the presence of telomeric C-bands in both chromosome arms, seven different types of anaphase I in respect to the constitution of their chromosome $1 \mathrm{R}$ (proceeding from cells in which wheat-rye pairing for this chromosome did not occurr) can be expected. Fig. 1 shows diagrammatically the different anaphase I types expected as well as the minimum number of chiasmata necessary for their appearance.

Now, if a bound arm at metaphase I corresponds to only one chiasma, the expected frequencies of the different anaphase I types can be estimated from metaphase I results (table 1): Type I would proceed from cells having a univalent pair; type II would proceed from cells with open bivalents with the long arm bound; type III would proceed from cells with open bivalents with the short arm bound and cells having ring bivalents would produce types IV, V and VI with the relative frequencies $1: 1: 2$, assuming that there is no chromatid chiasma interference across centromere.

50 anaphase I cells per plant were observed, examples are shown in plate 3. No evidence of recombination between wheat and rye chromosomes could be observed at this phase.

Table 2 shows the comparison between the observed and the expected frequencies of the different anaphase I types for chromosomes $1 \mathrm{R}$ under the assumption of a 1:1 correspondence between chiasmata and metaphase I bonds. The difference between observed and expected values is highly significant, an excess of anaphase I chromosomes showing no evidence of recombination being found.

The possibility of more than one chiasma per bond can also be considered: for a bivalent arm, if there is no chromatid chiasma interference, the frequency of anaphase I chromosomes showing evidence of recombination would be (Mather, 1935):

$$
F r=f 1+\frac{1}{2} f 2+\frac{3}{4} f 3+\cdots+\frac{2}{3}\left[1-\left(-\frac{1}{2}\right)^{n}\right] f n
$$

and the frequency of anaphase I chromosome arms showing no evidence of recombination:

$$
F p=f 0+\frac{1}{2} f 2+\frac{1}{4} f 3+\cdots+\frac{1}{3}\left[1-\left(-\frac{1}{2}\right)^{n-1}\right] f n
$$

in which $f 0, f 1, f 2, \ldots, f n$ are the frequencies of $0,1,2, \ldots, n$ chiasmata respectively. 
TABLE 2

The comparison between the observed and the expected frequencies of the different anaphase I types for chromosomes $1 R$ (see fig. 3) under the assumption of a 1:1 correspondence between chiasmata and metaphase I bonds. 50 anaphase I cells per plant were observed

\begin{tabular}{lllllllll}
\hline & & \multicolumn{7}{c}{ Anaphase I types } \\
\hline Plant & I & II & III & IV & V & VI & VII \\
\hline Che-1 & Obs. & 34 & 16 & - & - & - & - & - \\
& Exp. & 22 & 25 & 2 & $0 \cdot 25$ & $0 \cdot 25$ & $0 \cdot 5$ & 0 \\
CHE-2 & Obs. & 35 & 15 & - & - & - & - & - \\
& Exp. & 22 & 23 & 1 & 1 & 1 & 2 & 0 \\
CHE-3 & Obs. & 32 & 18 & - & - & - & - & - \\
& Exp. & 23 & 21 & - & $1 \cdot 5$ & $1 \cdot 5$ & 3 & 0 \\
CHE-4 & Obs. & 37 & 13 & - & - & - & - & - \\
& Exp. & 22 & 24 & 3 & $0 \cdot 25$ & $0 \cdot 25$ & $0 \cdot 5$ & 0 \\
\hline \multirow{2}{*}{ Total } & Obs. & 138 & 62 & - & - & - & - & - \\
& Exp. & 89 & 93 & 6 & 3 & 3 & 6 & 0 \\
\hline
\end{tabular}

Contingency $\chi^{2}$ between observed and expected values in totals (anaphase I types III, IV, V and VI have been added): $\chi^{2}=34 \cdot 777 ; 0.001>p$.

If

$$
R=\frac{1}{2} f 2+\frac{3}{4} f 3+\cdots+\frac{2}{3}\left[1-\left(-\frac{1}{2}\right)^{n}\right] f n
$$

and

$$
P=\frac{1}{2} f 2+\frac{1}{4} f 3+\cdots+\frac{1}{3}\left[1-\left(-\frac{1}{2}\right)^{n-1}\right] f n
$$

we can rewrite Mather's equations as:

$$
F r=f 1+R ; \quad F p=f 0+P .
$$

$f 0$ can be estimated from the metaphase I results since it equals the frequency with which the arm considered is not bound. $F r$ and $F p$ can be estimated from the anaphase I observations.

Now, if a maximum of two chiasmata per arm are formed, $P=R=\frac{1}{2} f 2$, and in this case $f 1$ and $f 2$ can be deduced.

Table 3 shows the estimated values of $f 0, f 1$ and $f 2$ for each $1 \mathbf{R}$ chromosome arm in the plants studied under the assumption of a maximum of two chiasmata per arm (in these estimates, $1 \mathrm{R}$ chromosome arms involved in wheat-rye associations have been considered as not bound arms). The estimates lead to the absurdities of a negative value of $f 1$ in the short arm and to a lower value of $f 1$ than $f 0$ or $f 2$ in the long arm.

If more than two chiasmata per arm were formed no estimates of $f 1, f 2, \ldots, f n$ can be made, however, in this case $R>P$, and the value of $f 1$ would be lower than the one estimated under the assumption of a maximum of two chiasmata per arm (table 3), the absurdity being increased.

\section{Discussion}

The excess of anaphase I parental chromosomes found in the meiosis of the Triticale-rye hybrids analysed in this work do not fit the expectation 
TABLE 3

The estimated $\left(^{*}\right)$ frequencies of $0(f 0), 1$ (f1) and 2 (f2) chiasmata in the two arms of chromosome $1 R$, under the assumption of a maximum of two chiasmata per arm

\begin{tabular}{lccccccc}
\hline & \multicolumn{3}{c}{ Short arm } & & & \multicolumn{3}{c}{ Long arm } \\
\cline { 2 - 3 } \cline { 6 - 8 } Plant & $f 0$ & $f 1$ & $f 2$ & & $f 0$ & $f 1$ & $f 2$ \\
\hline CHE-1 & 0.94 & -0.06 & 0.12 & & 0.48 & 0.12 & 0.40 \\
CHE-2 & 0.90 & -0.10 & 0.20 & & 0.46 & 0.06 & 0.48 \\
CHE-3 & 0.88 & -0.12 & 0.24 & & 0.46 & 0.18 & 0.36 \\
CHE-4 & 0.92 & -0.08 & 0.16 & & 0.50 & 0.02 & 0.48 \\
\hline Total & 0.91 & -0.09 & 0.18 & & 0.475 & 0.095 & 0.43 \\
\hline
\end{tabular}

${ }^{*}$ ) $f 0=$ frequency of not bound bivalent arms at metaphase I (table 1 ). (1R chromosome arms involved in wheat-rye associations have been considered as not bound arms). $f 2=$ $2(F p-f 0) ; F p=$ frequency of anaphase I cells showing no evidence of recombination in the chromosome arm considered (table 2 ). $f 1=1-f 0-f 2$.

neither under the hypothesis of one bond-one chiasma (table 2) nor under the possibility of more than one chiasma per bond being formed (table 3 ).

On the other hand, although the data could be explained in part with the existence of chromatid chiasma interference (i.e., all bonds in the short arm and most bonds in the long arm being the result of two chiasmata in which the same two chromatids are involved), a too complicated picture would result and therefore this possibility can certainly be excluded.

The existence of non-chiasmate bonds proposed earlier (Orellana and Giraldez, 1983) in desynaptic rye would easily explain an excess of anaphase I parental chromosomes when compared to the frequency with which a given chromosome arm is not bound at metaphase I. These non-chiasmate bonds would represent a remnant of prophase pairing that could be similar to the process found in achiasmate organisms in which chromosomes remain paired till anaphase I onset (see Baker et al., 1976). The existence of nonchiasmate associations at metaphase $I$ in organisms that do form chiasmata has also been claimed by John (1976) and King and John (1980) as an explanation for the terminal chromosome associations found in grasshoppers.

Under this point of view, a bivalent arm can have three different possibilities at metaphase I: it can be bound having at least one chiasma (with a probability $C B=f 1+f 2+\cdots+f n$ ), it can be bound having no chiasmata (with a probability $N C B$ ) or it can be not bound (with a probability $N B$ ). In this case, the relationships between anaphase I and metaphase I results would be:

$$
\begin{aligned}
& F r=f 1+\frac{1}{2} f 2+\frac{3}{4} f 3+\cdots+\frac{2}{3}\left[1-\left(-\frac{1}{2}\right)^{n}\right] f n \\
& F p=N B+N C B+\frac{1}{2} f 2+\frac{1}{4} f 3+\cdots+\frac{1}{3}\left[1-\left(-\frac{1}{2}\right)^{n-1}\right] f n .
\end{aligned}
$$

Cytologically, only two possibilities can be distinguished at metaphase I, bound arms $(B=C B+N C B)$ and not bound arms $(N B)$. So, only if a maximum of one chiasma is formed in the bivalent arm considered the frequency with which non chiasmate bonds $(N C B)$ are formed can be deduced. 
In the hybrids analysed here this is probably the expected situation, given the low number of bonds formed when compared to normal rye. In this case all bonds formed in the short arm of bivalent $1 R$ would be nonchiasmate while in the long arm non-chiasmate bonds would appear with a probability $N C B=0 \cdot 215$.

This value can be compared with the one obtained in an earlier work (Orellana and Giraldez, 1983) for the same chromosome arm in desynaptic plants of rye (B1PEE). In that instance the frequency with which the long arm of bivalent $1 \mathrm{R}$ was not bound at metaphase I was $N B=0.0225$ and the frequency of anaphase I parental chromosomes was $F p=0 \cdot 2072$. The probability of non-chiasmate bonds assuming that a maximum of one chiasma was formed was $N C B=F p-N B=0 \cdot 1847$.

In the present work a higher frequency of unbound arms at metaphase I $(N B=0.475)$ is accompanied by a higher frequency of non-chiasmate bonds. This can be explained if we consider that in these hybrids chiasma formation is more severely affected than bound arm formation, i.e., the proportion of metaphase I bonds that are the result of one chiasma is lower in these hybrids than in the desynaptic plants mentioned above, the frequency of non-chiasmate bonds being increased.

The results obtained in the Triticinae concerning interspecific gene transference by means of meiotic recombination support the existence of chiasmata between chromosomes of the different species involved. However, in this work no evidence of recombination between chromosomes of wheat and rye has been observed. If the existence of non-chiasmate bonds is extended to homoeologous metaphase I bonds, the frequency of interspecific recombination in Triticinae can be smaller than the one estimated from cytological observations at metaphase I if a minimum of one chiasma per bond is considered.

Acknowledgements. This work has been partially supported by a grant from the Comision Asra de Investigación Científica y Técnica of Spain.

\section{REFERENCES}

BAKER, B. S., CARPENTER, A. T. C., ESPOSITO,M. S., ESPOSITO R. E. AND SANDLER, L. 1976. The genetic control of meiosis. Ann. Rev. Genet., 10, 53-134.

GIRALDEZ, R., CERMEÑO, M. C. AND ORELLANA. J. 1979. Comparison of C-banding pattern in the chromosomes of inbred lines and open pollinated varieties of rye. $Z$. Pflanzenzuchtg., $83,40-48$.

GIRALDEZ, R. AND ORELLANA, J. 1979. Metaphase I bonds, crossing over frequency, and genetic length of specific chromosome arms of rye. Chromosoma, 72, 377-385.

JOHN, B. 1976. Myths and mechanisms of meiosis. Chromosoma, 54, 295-325.

KING, M. AND JOHN, B. 1980. Regularities and restrictions governing C-band variation in Acridoid grasshoppers. Chromosoma, 76, 123-150.

MATHER, K. 1935. Reductional and equational separation of the chromosomes in bivalents and multivalents. J. Genet., 30, 53-78.

NARANJO, T. 1982. Preferential occurrence of wheat-rye meiotic pairing between chromosomes of homoeologous group 1. Theor. Appl. Genet., 63, 219-225.

ORELLANA, J. AND GIRALDEZ, R. 1981. Metaphase I bound arms and crossing over frequency in rye. I. Open pollinated varieties. Chromosoma, 84, 439-449.

ORELLANA, J. AND GIRALDEZ, R. 1983. Metaphase I bound arms and crossing over frequency in rye. III. Non-chiasmate bonds in desynaptic plants. Heredity, 51, 383-394. 